\title{
«VOUS NE SEREZ JAMAIS BIEN LOIN DE LA MER.» SOUVENIRS ROUMAINS DES ÉDITIONS DE L'HERNE, À PARIS, À LA CHARNIÈRE DES ANNÉES 80 ET 90
}

\author{
"YOU WILL NEVER BE VERY FAR FROM THE SEA". \\ ROMANIAN MEMORIES FROM THE HERNE PUBLISHING \\ HOUSE, PARIS, AT THE END OF THE 1980'S
}

\section{Sophie ROCHEFORT-GUILLOUET ${ }^{1}$}

https://doi.org/10.52744/9786062613242.09

\begin{abstract}
Résumé : Ce texte rappelle mes souvenirs de Cioran, que j’ai eu le privilège de côtoyer, à l'époque où je travaillais aux Éditions de l'Herne, en 1989. Cioran avait choisi un éditeur roumain pour publier les textes de jeunesse qu'il avait écrits dans sa langue maternelle, avant de venir vivre à Paris. Cette période, où il rencontrait Rue de Verneuil des amis et des connaissances de la communauté roumaine du monde des lettres et des arts en exil, fut particulièrement riche car elle correspond à celle de la révolution qui, à Bucarest, mit alors un terme à l'ère communiste et au pouvoir de Ceausescu.
\end{abstract}

Mots-clés : Édition ; Littérature ; Culture franco-roumaine ; Traduction ; Révolution.

\begin{abstract}
This text recalls memories from the years I spent working in Paris for a publishing house, les Éditions de l'Herne, in a position which gave me the opportunity and the privilege to meet Cioran. He had chosen to publish there the translation of the works he wrote in his native tongue, in his early years, before he left to come and settle in France. At that time, the bookshop located on Rue de Verneuil was a beehive, the place where artists and writers from the Romanian diaspora would come and share. Topics were discussed in a Franco-Romanian idiom with an enthusiasm fuelled by the very events taking place at that same time in Bucharest when communism collapsed and Ceausescu was removed.
\end{abstract}

Keywords: Publishing; Literature; French-Romanian Culture; Translation; Revolution

\footnotetext{
${ }^{1}$ Enseigne l'Histoire comparée de l'Europe et de l'Asie et l'Histoire de l'Art à SciencesPo Paris ainsi que la Littérature à l'Université de Rouen, France. sophie.rochefortguillouet@sciencespo.fr
} 
Il y avait une cave voûtée remplie de livres mais aussi des étagères, surchargées de volumes, dans une pièce minuscule, unique, qui servait à la fois de librairie et de maison d'édition. Rue de Verneuil. Pendant quelques années, les Éditions de l'Herne furent au cœur de ma vie. J'étais normalienne, en Lettres classiques, donnant des cours à la Sorbonne, en suivant d'autres rue d'Ulm et à Sciences-Po, me dispersant dans le tourbillon littéraire qui vous saisit au terme de deux ans de classe préparatoire. J'avais envoyé une candidature aux Éditions de l'Herne et reçu très vite une réponse encourageante du directeur, Constantin Tacu, qui m'avait donné rendez-vous pour discuter des modalités d'un travail à mi-temps.

Il m'a reçue au milieu de cartons - un envoi un peu en retard du dernier Cahier aux souscripteurs - et m'a donné un exemplaire de l'ouvrage paru, sans savoir qu'il allait rejoindre une collection déjà importante de volumes édités par ses soins, allant de Lovecraft à Poe, de Jung à Char et à bien d'autres, trouvés ou bien au prix fort chez Gibert ou bien avec bonheur, soldés, chez les bouquinistes. Celui-ci était consacré à Hölderlin. Il m'a dit : « Vous verrez Cioran prochainement, je vais éditer ses premières œuvres, encore écrites en roumain avant qu'il ne vienne en France et ne devienne l'auteur de De l'inconvénient d'être né », puis il a prononcé le titre de l'ouvrage à venir : Sur les cimes du désespoir. Nous avons parlé de Cleobis et de Biton, ces deux éphèbes à qui Zeus octroya la mort, à la demande de leur mère qui requérait pour eux le meilleur don des Dieux. J'ai commencé à travailler la semaine suivante. Qui aurait pu décliner pareille offre ? Et je me suis demandé à quoi ressemblerait une rencontre avec Cioran.

Les Éditions de l'Herne, aux alentours de 1989, étaient une sorte de cercle littéraire, de café artistique et politique, l'épicentre du monde roumain en exil à Paris. Au début, derrière mon bureau, occupée à des tâches de secrétariat et de relecture de manuscrits, je voyais passer des écrivains, des artistes, des critiques, des journalistes, qui parlaient roumain avec Constantin et me disaient bonjour en français. Peu à peu, nous commençâmes à discuter de Brancusi dont l'atelier allait devenir un centre d'intérêt majeur du Musée d'Art Moderne de Beaubourg. Je me souviens particulièrement de L'oiseau dans l'espace et de Mademoiselle Pogany, Constantin en parlait les yeux fermés, décrivant chaque volume et chaque courbe, en accompagnant ses mots d'une volte de la main. Edgar Reichmann était critique littéraire et écrivain, il passait au moins une fois par semaine et évoquait pour nous en avant-première son prochain article dans Le Monde. Georges Banu parlait cinéma, détaillait l'avancée de son Cahier sur le Théâtre et l'opéra, lieux de Mémoire. 
Mariana Sora travaillait à un petit ouvrage d'entretiens avec Cioran. Bientôt la Révolution allait éclater à Bucarest, chasser le Conducător et faire accéder au pouvoir des gens connus du cénacle parisien. On suivait les informations à la radio. La Securitate, Timisoara. Puis, Iliescu et Roman. Lorsque parut l'Éthique de Robinson d'Andrei Plesu parmi les auteurs de l'Herne, celui-ci était déjà devenu Ministre de la culture. On s'interrogeait sur ce qu'Eugène Ionesco pensait de tout cela. Cioran rappelait ses souvenirs de l'année 1928, lorsque lui-même, Eliade et Ionesco apprenaient le français à Bucarest. Je découvrais l'existence d'un Petit Paris au cœur de la capitale roumaine. On se demandait aussi ce qu'il adviendrait des jeunes éditions libres, Humanitas, et de son somptueux catalogue. Très vite, elles publieraient les auteurs interdits sous Ceausescu : Cioran, Eliade, Lovinescu, Ierunca.

Il y avait aussi Alex dont je n'ai jamais connu, je crois, le nom de famille, qui s'installait dans un vieux fauteuil en cuir et parlait de Pierre Restany, d'Yves Klein, des Nouveaux Réalistes, des aquarelles qu'ils vendaient et du jour où il pourrait retourner en Roumanie. Il évoquait Sultana Maïtec avec Constantin. Elle avait préparé une lithographie pour accompagner la sortie du petit volume d'entretiens avec Cioran. Sur le mur de mon bureau, une feuille d'or ronde, solaire, me rappelle aujourd'hui encore son art, avec, sur le mur opposé, un visage blanc lunaire, sans bouche. Constantin travaillait à l'édition de textes précoces de Mircea Eliade. Il avait dirigé le cahier qui lui fut consacré. Le catalogue s'étoffait de mois en mois : L'Inde, Journal des Indes, Océanographie, Alchimie Asiatique et surtout, un peu plus tard, un texte fondateur : Commentaires sur la légende de Maître Manole (Commentarii la lengenda Mesterului manole). "On oublie souvent », écrivait Eliade, « que les Roumains et leurs voisins du Sud-Est de l'Europe ont retrouvé leur destin dans le mythe central de la mort créatrice ». Je connaissais ce conte terrible d'amour et de sacrifice. J'avais lu avec passion les œuvres de Marguerite Yourcenar et notamment les Nouvelles Orientales qu'elle composa en 1938. Le recueil comporte un texte sombre, glaçant comme un mythe, intitulé « Le lait de la mort », inspiré d'une légende albanaise, Le Château de Rozafa. La construction d'un bâtiment exige inéluctablement la mort d'une jeune femme, enfermée dans le mur, scellée dans le mortier.

Et un jour, Cioran était donc enfin entré dans la libraire. Je me demandais chaque matin si ce serait le jour finalement... Je ne l'ai pas entendu, je n'ai levé les yeux que lorsqu'il s'est manifesté devant mon bureau. L'ordinateur attirait toujours un regard de suspicion de sa part. Il m'a demandé s'il était en avance car Constantin n'était pas encore arrivé ce matin-là. Aucun doute, il ressemblait à toutes ces photos vues et revues 
sur les jaquettes et dans les anthologies, mais il a souri et cela m'a semblé étonnant. J'allais découvrir qu'il savait être drôle à sa manière, et parfaitement tragique en même temps, quand il parlait de son enfance. Il y a longtemps qu'il n'avait plus de prénom en France, Emile Michel, juste un nom prononcé en français à la diable, plein de voyelles, mais qui claquait dans les lectures de ceux dont les nuits sont tourmentées. Bréviaire des insomniaques. Vade mecum des amoureux de Bach et de tous ceux qui croient qu'on peut encore discuter d'égal à égal avec Dieu. Une telle solitude, titanesque, forçait le respect. « On ne lit pas Cioran, me disait mon professeur de philo au lycée, il lit en vous et cela fait peur ». J'avais appris par cœur des aphorismes, ceux qui vous font croire que vous avez compris le maître et que vous partagez avec lui la connaissance secrète de ce que la vie ne vaut pas la peine d'être vécue. On est bien jeune avant d'être vieux... Chez moi, Cioran était rangé à côté de Byron, de Baudelaire et de Schopenhauer, je n'ai jamais vraiment cru au classement par genres, langues ou thèmes. Ces quatre-là entretenaient de secrètes affinités, un quant-à-soi qui plaisait à ma propre solitude studieuse, un noli me tangere qui permettait de rester au-dessus de la mêlée. Peut-on réellement classer les auteurs selon leur degré d'élégance désabusée ? De détachement, de mépris des choses et des pensées triviales ? Vêtement sobre, pardessus, cheveux blancs en arrière comme si le vent avait été débout sur le trajet qui le menait de l'Odéon à la rue de Verneuil, Cioran entrait, marchait un peu dans la librairie, regardait les tranches des livres puis s'asseyait. J'imaginais dans l'avenir des agapes aux Enfers, mon quatuor de lettres infernal disséquant le Monde, l'Histoire, la littérature, la gloire, en se souriant à demi, en sachant que le jeu dépasse souvent l'acteur et donne vie au personnage de légende. Et tous riraient de la vanité des vivants.

Cioran était donc là. Il s'enveloppait de silence ou parlait à voix basse, cherchant le mot juste. On aurait dit qu'il avait un compte ouvert, mais limité, dans le dictionnaire et qu'il ne voulait pas l'épuiser trop vite. Il arrivait de la rue de l'Odéon, il parlait de Dieppe et de la mer, des promenades ventées sur la jetée. Il s'amusait des notables qui préféraient la cossue Varengeville pour des weekends entre soi. Il évoquait la pluie grise mais parlait sans cesse des teintes nuancées que prend la mer le long de la côte normande lorsque le soleil frappe les falaises blanches. Parfois, il secouait la tête et le mutisme le prenait. Constantin arrivait, Cioran s'animait et tous deux parlaient livres et traductions, créant un idiome où deux langues se mêlaient le plus souvent à part égale. Puis ils partaient déjeuner, retrouver un écrivain de passage. Le quartier regroupait la crème du monde de l'édition. Gallimard avait les droits sur les livres de Cioran un pavé d'œuvres complètes - mais Constantin avait son amitié. Lorsque la 
traduction française de Sur les Cimes du désespoir fut prête, lorsque le choix de la couverture fut validé, lorsqu'il l'imprimeur livra des caisses de livres pour le service de presse, quelque chose se passa. Une réticence. Il aurait fallu dédicacer certains volumes afin de les envoyer aux journalistes des pages littéraires des grands quotidiens, des revues, des magazines, aux professeurs qui déjà s'étaient spécialisés dans le grand pessimiste. Une lassitude saisit Cioran. Je ne l'ai jamais entendu critiquer ou dénigrer. Son univers était situé au tout début de la création littéraire car rien n'est beau que les commencements, défiant la postérité, alors à quoi bon une signature, une faveur enrubannée quand le livre est déjà accouché. On trouva un stratagème qui lui permit de ne signer qu'une dizaine d'exemplaires : un joli carton crème, commandé chez un grand imprimeur $\mathrm{du} 7^{\mathrm{e}}$ arrondissement, un " prière d'insérer » sous forme d'excuse et de pirouette : "L'auteur, absent de Paris, ne pouvait signer son œuvre et espérait qu'on voudrait l'en excuser ». Pendant un court instant, il y eut de la malice dans le regard du maître en désespoir. Le jour où il vint signer les ouvrages, il écrivit un trésor pour moi, « Pour Sophie, afin qu'elle sache le plaisir que j'ai à m'entretenir avec elle ». Cioran, sa signature commence ferme et lisible puis le $\mathrm{r}$ penche dangereusement et la finale plonge.

Une phrase est restée plus que d'autres dans ma mémoire, nous l'avions choisie avec Constantin pour la quatrième de couverture : " J'aimerais vivre au commencement du monde, dans le vortex démonique des turbulences primordiales. Que tout vibre d'un frémissement primordial, tel un éveil du néant ». André Vornic avait eu la charge de traduire Cioran du roumain en français, une tâche ardue dont il avait fait un court chef d'œuvre de style économe, sous la relecture attentive de la philosophe Christiane Frémont. Le livre grondait, on aurait dit qu'il contenait la matrice d'un monde en gésine, il débordait. Il avait été rédigé en 1934, sous le titre Pe culmine disparari. Cioran avait écrit un cours texte d'introduction pour expliquer la formule et le contexte : " J'ai écrit ce texte à l'âge de 22 ans dans une ville que j'aimai, Sibiu, en Transylvanie ». Il travaillait alors à une thèse et rassuraient ses parents en évoquant des études potentielles de philosophie. Il ne dormait que très peu et marchait, marchait sans fin, surtout la nuit. Il croisa par hasard un article de journal racontant le triste suicide d'un désespéré. La manchette évoquait un homme irrémédiablement perdu " sur les cimes du désespoir ». La formulation hyperbolique lui sembla être judicieuse, en résonance avec son expérience intime. Un autre ouvrage Des larmes et des saints, publié en Roumanie en 1940 sous le titre Lacrimi si sfinti, parut au catalogue des Editions de l'Herne. Il fut préfacé par Sanda Stolojan qui publia à la même époque un étonnant récit du voyage en Roumanie, en 1968, du Général de Gaulle, dont elle fut l’interprète. 
La vie en Roumanie avant de gagner la France était au cœur des conversations dans le bureau. Constantin, qui avait dû partir pour fuir le communisme, lançait un souvenir, le développait, Cioran le reprenait. Avons-nous vraiment un jour discuté de la manière dont les paysans castraient les poulains avant-guerre ? Comment en étions-nous arrivés à discuter des épreuves de russe au lycée de Bucarest, épreuves dont Constantin rappelait que tout le monde faisait semblant et qu'il suffisait d'apprendre par cœur un poème de Pouchkine pour obtenir satisfecit ? Par quels méandres de la conversation avons-nous comparé ensemble des recettes de polenta ? J'ai découvert la Ciorba et les légendes, les blagues tristes de Cioran sur les Roumains, sur les Allemands, sur les Hongrois. Nous avons récité des poèmes ensemble de Tristes et des Pontiques d'Ovide en déplorant que l'auteur de L'art d'aimer n'ait jamais pu se faire à cet exil dace auquel l'empereur Auguste le condamna. Comment ne pas se plaire à Constanța même dans l'Antiquité ? Dès qu'il y a la mer... on peut survivre. Je leur parlais de Saint Malo. Suave mari magno disait Constantin. Cioran acquiesçait. Constantin avait la fibre latine, il connaissait les auteurs, les historiens, et aimait les noms d'empereurs et de poètes romains que portaient ses contemporains. Cioran souriait, indulgent, nous étions trop enthousiastes. Et on finissait par aller manger un bœuf gros sel dans le quartier.

Il y avait aussi de l'ombre derrière cet héritage, une histoire complexe, des tensions et des enjeux qui n'avaient pas attendu le communisme pour sourdre, des persécutions. On parlait des textes de Malaparte sur la Seconde Guerre Mondiale et sur les déportations des juifs. Les gardes de fer de Codreanu, la prise de pouvoir d'Ion Antonescu, des figures sombres s'invitaient soudain dans le débat. Cioran a vécu dans le siècle, il n'a jamais été en marge de l'Histoire ou indifférent. Il y avait enfin la musique quand les sujets devenaient oppressants et l'ombre tutélaire d'Enesco planait, cet éternel voyageur entre la Roumanie et Paris. " Pour le comprendre, Sophie », disait Cioran, « il faut savoir ce que sont les doinas roumaines, ces courts poèmes ». Et il expliquait, citait. " Certes, mais il faut aussi savoir danser les horas roumaines », concluait gravement Constantin qui se lançait alors dans une description sérieuse de chorégraphies circulaires.

En 2009, un Cahier de l'Herne fut consacré à Cioran, dirigé par Laurence Tacu. La célèbre couverture blanche comporte toujours une photo en noir et blanc qui reste pour moi le meilleur rappel de l'écrivain que j'ai connu. Un pull-over, une veste de tweed, un pardessus fané dans un coin, le regard lointain comme ennuyé de poser une fois encore, la tête penchée. Sur fond de livres. J'ai quitté l'Herne avec tristesse pour aller vivre plusieurs années à Londres. Lorsque j'ai annoncé mon départ à 
Cioran, il m'a demandé de lui donner un exemplaire du Crépuscule des pensées, le dernier volume publié de ses textes de jeunesse (Amurgul gândurilor), publié en roumain alors qu'il vivait depuis dix ans à Paris. Il a écrit une dédicace, refermé le volume et m'a dit, avant de partir : " Làbas au moins vous ne serez jamais loin de la mer ». Il avait écrit " pour tant regrettée Sophie » et souligné deux fois le mot tant. Une expérience sans commune mesure venait de prendre fin. Constantin a souri et m'a dit doucement : "Vous le reverrez à votre retour, et de toute façon, il est immortel, ni Dieu ni le Diable ne voudront de lui ! Ils n'ont pas assez de conversation... »

Lorsque j'ai poussé à nouveau la porte de la Rue de Verneuil, en juillet 1995, Cioran avait quitté la scène pour toujours depuis un mois déjà. Puis, Constantin Tacu est parti à son tour. Il reste une photo extraordinaire des deux hommes, marchant côte à côte dans le quartier. Lorsque l'Université Cantemir m'a invitée en décembre 2020, à l'occasion d'un colloque, j'ai enfin arpenté les rues de Bucarest en pensant que, grâce à eux deux, je visitais un pays familier, je déambulais dans une ville que j'avais déjà connue un peu dans une autre vie.

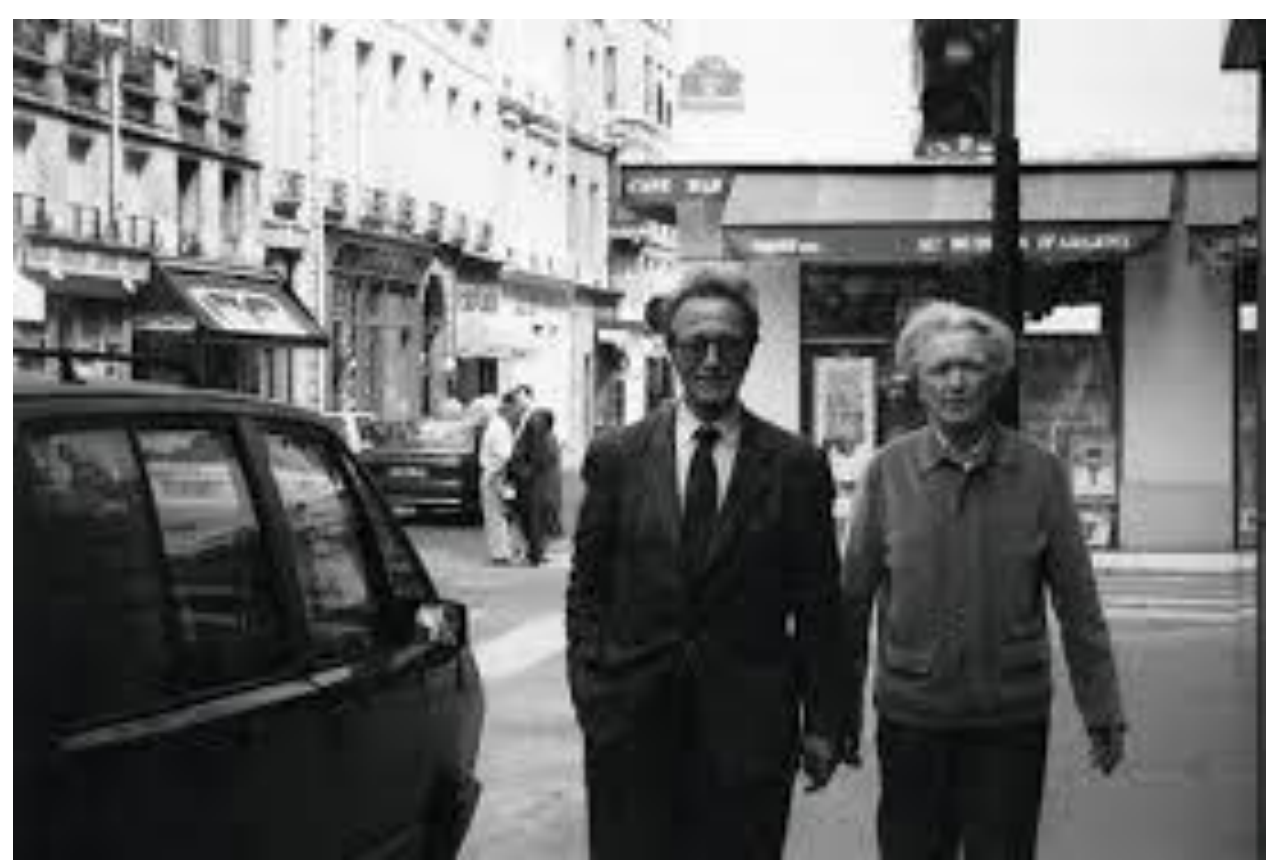

